\title{
Progress of Liposomal Nanohybrid Cerasomes as Novel Drug Nanocarriers
}

\author{
Shihao Li, ${ }^{a, b}$ Jie Jiang, ${ }^{a, b}$ Shanshan Zhu, ${ }^{b}$ Yinan Yan, ${ }^{b, c}$ Gang Huang, ${ }^{*, d}$ and Dannong $\mathrm{He}^{\star, a, b}$ \\ ${ }^{a}$ School of Materials Science and Engineering, Shanghai Jiao Tong University, Shanghai 200240, China \\ ${ }^{b}$ National Engineering Research Center for Nanotechnology, Shanghai 200241, China \\ ${ }^{c}$ Key Laboratory of Synthetic and Self-Assembly Chemistry for Organic Functional Molecules, \\ Shanghai Institute of Organic Chemistry, Chinese Academy of Sciences, Shanghai 200032, China \\ ${ }^{d}$ Shanghai University of Medicine \& Health Sciences, Shanghai 201318, China
}

\begin{abstract}
Despite many benefits, liposomes have still not realized their full potential as vehicles for drug delivery due to the morphological instability. Recently, liposomal nanohybrid cerasomes have been developed as novel drug nanocarriers based on organoalkoxysilane through a sol-gel reaction in combination with self-assembly process. The presence of polyorganosiloxane network on the surface imparts cerasomes higher morphological stability than conventional liposomes and the incorporation of liposomal bilayer structure into cerasomes boosts the biocompatibility in comparision with silica nanoparticles with similar size. Moreover, cerasomes are able to encapsulate various drug molecules and exhibit controlled drug release profile. In addition, cerasomes are easy to be conjugated with biomolecules through silane-coupler chemistry due to the silanols on the surface. Therefore, cerasomes are expected to be ideal drug delivery systems owning to the unique advantages. The present paper will briefly introduce the preparation and properties of cerasomes, followed by reviewing the progress of cerasomes for drug delivery.
\end{abstract}

Keywords cerasomes, cerasome-forming lipids, preparation, properties, drug delivery

\section{Introduction}

Nowadays, chemotherapy is an important means for cancer treatment. ${ }^{[1]}$ Despite many benefits, patients receiving anticancer drugs often suffer unfavorable side effects due to the non-specific toxicity of anticancer drugs. Most anticancer drugs can kill cancer cells with non-selective killing of the normal human cells, which brings great pains to patients. In order to overcome this problem, drug delivery systems (DDS) based on nanotechnology have been widely developed. ${ }^{[2-4]}$ To a certain extent, nano-DDS can solve many problems associated with drug molecules like low drug solubility, fast clearance rates, non-specific toxicity, thereby enhancing therapeutic efficiency and reducing side effects. ${ }^{[5,6]}$ Common DDS include liposomes, ${ }^{[7-9]}$ mesoporous silicon, ${ }^{[10-12]}$ polymer micelles, ${ }^{[13-15]}$ hydrogels. ${ }^{[16,17]}$ Among them, liposomes have received widespread attention due to their biocompatibility, degradability and ability to encapsulate various drugs. ${ }^{[18]}$ However, liposome-based drug carriers have not attained their full potential due to their insufficient morphological stability, which has limited their clinical applications. ${ }^{[19,20]}$

Recently, a novel type of organic-inorganic bilayer vesicle named "cerasome" has been developed in the field of drug delivery. ${ }^{[21]} \mathrm{Ce}-$ rasomes with liposomal bilayer structure are biomimetic colloidal nanoparticles covered with the additional polyorganosiloxane surface, which imparts cerasomes much higher stability than conventional liposomes. ${ }^{[22]}$ Besides, cerasomes have better biocompatibility than silica nanoparticles with similar size due to the presence of liposomal structure. ${ }^{[23]}$ Cerasomes integrate the advantages of both liposomes and silica nanoparticles but overcome their shortings. In addition, cerasomes can encapsulate a wide range of drug molecules and the silanols on the surface make it convenient for cerasomes to be functionalized to effectively delivery drugs to desired areas via silane- coupler chemistry. ${ }^{[24]}$ Therefore, cerasomes, serving as new drug delivery carriers, possess great potential for clinical applications due to their unique advantages.

This paper will summarize the progress of liposomal nanohybrid cerasomes and their applications as drug nanocarriers.

\section{Overview of cerasomes}

In 1999, Katagiri et al. ${ }^{[21]}$ designed and prepared a novel organicinorganic nanohybrid cerasome successfully based on an organoalkoxysilane with a lipid-like structure through a sol-gel reaction in combine with self-assembly process. Afterwards, numerous morphologically stable cerasomes have been fabricated by employing various artificially designed cerasome-forming lipids (CFL). ${ }^{[23]}$ As shown in Figure 1, CFL consists of a hydrophilic triethoxysilyl head moiety, a hydrophobic alkane chain segment and a connector unit between them. ${ }^{[25]}$ When the triethoxysilyl groups are hydrolyzed to form silanol groups under appropriate conditions, cerasome-forming lipids become amphiphilic molecules, followed by self-assembly to form lipid bilayer vesicles. Then the silanol groups on the surface of vesicles are condensed to form polyorganosiloxane network, resulting in the formation of liposomal nanohybrid cerasomes. What's more, cerasomes can be loaded with hydrophilic, hydrophobic or amphiphilic drug molecules and exhibit sophisticated controlled release behavior. ${ }^{[26-28]}$ For example, Cao et al. ${ }^{[27]}$ prepared successfully hydrophobic drug paclitaxel (PTX)-loaded cerasomes with higher morphological stability and controlled sustained drug release in comparision with liposomes, which demonstrated for the first time that liposomal nanohybrid cerasomes could be a promising drug delivery system.

* E-mail: huang2802@163.com,hdn_nercn@163.com Received September 8, 2017; accepted October 28, 2017. 


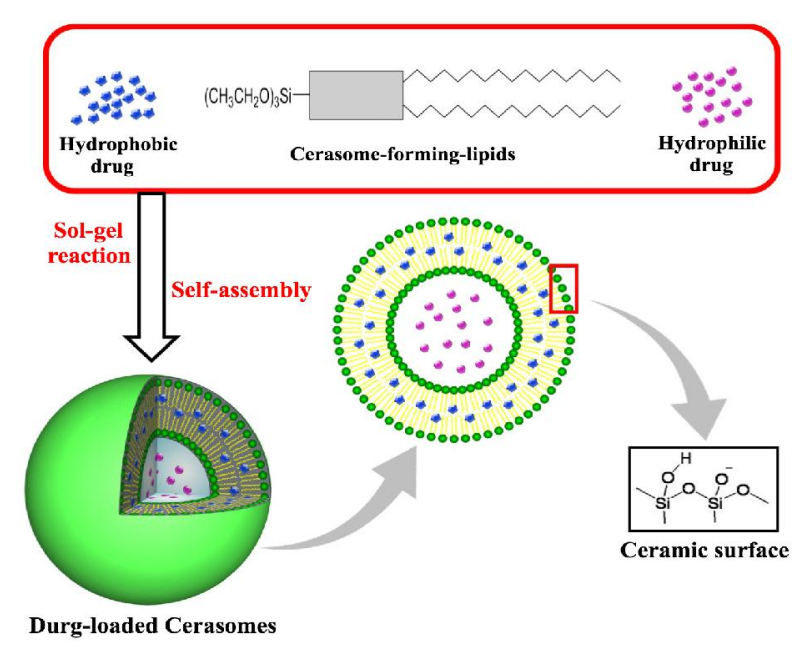

Figure 1 General molecular structure of a cerasome-forming lipid and preparation schematic of drug-loaded cerasomes.

\section{Synthesis and properties of CFL}

Common CFL having both an organic and an inorganic part connected to each other in the molecule are shown in Figure 2. CFL 1 was synthesized through simple condensation reactions by using three molecular units, dihexadecyamine, succinic anhydride, and 3-aminopropyltriethoxylsilane. ${ }^{[29]}$ CFL 2 with a simple molecular structure having one urea group was obtained by one-step condensation of dihexadecylamine with 3-triethoxysilyl-propylisocyanate. ${ }^{[30]}$ CFL 3 having one amide group in the connector unit was prepared by hydrosilylation of triethoxysilane with $N, N$-dihexadecyl-3-butenamide, which was obtained by condensation of dihexadecylamine with 3-butenoic acid. ${ }^{[22]}$ CFL 1-3 were water-insoluble lipids and the triethoxysilyl head groups must be hydrolyzed to form amphiphilic molecules before further self-assembly. Cationic cerasome-forminglipids $\mathbf{4}$ and $\mathbf{5}$ with analogous structure to the typical peptide lipids were also prepared. CFL $\mathbf{6}$ with two triethoxysilyl head groups was engineered to regulate the density of siloxane framework on the cerasome surface. CFL 7 based on phospholipid with additional triethoxysilyl head group made it possible to prepare biodegradable cerasomes.

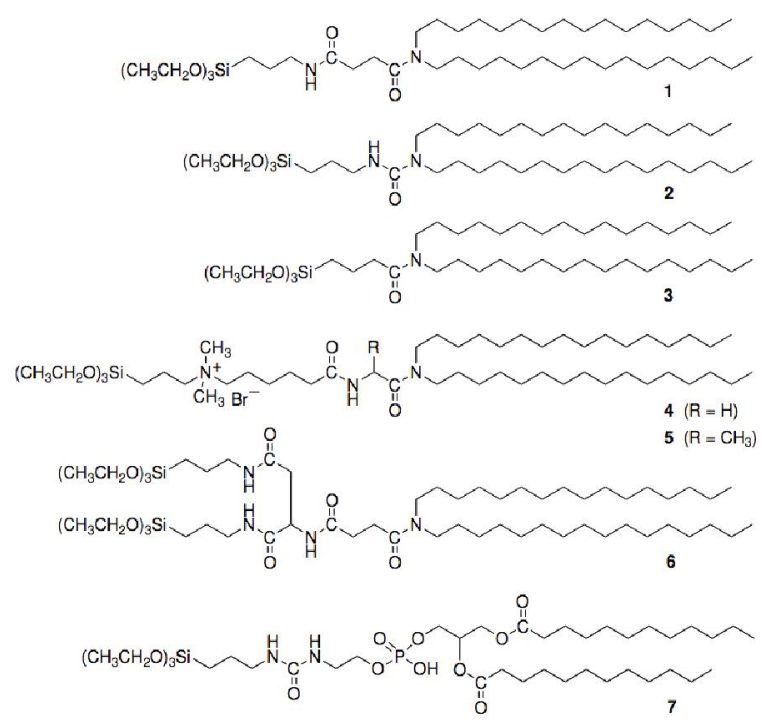

Figure 2 Structural formulae of cerasome-forming lipids.

\section{Preparation of cerasomes}

The preparation procedure of cerasomes can refer to that of liposomes to a certain extent due to the analogous vesicle structure between them. At present, three typical methods are adopted for the preparation of cerasomes, including ultrasonic dispersion method, ${ }^{[21]}$ ethanol sol injection method ${ }^{[22,24]}$ and thin film hydration method. $^{[28,31]}$

\section{Ultrasonic dispersion method}

The ceresome-forming-lipids and hydrochloric acid solution with an appropriate $\mathrm{pH}$ were firstly mixed and then vortex mixing until turbid dispersion was obtained, reflecting the formation of multi lamellar vesicle (MLV). Upon sonication with a probe-type sonicator, the turbid dispersion decreased to become clear, indicating the formation of smaller nanoparticles. The stable cerasome suspension was finally acquired after incubation of the transparent solution at room temperature overnight.

Obviously, this method is quite simple, however, it is undeniable that $\mathrm{pH}$ conditions play a key role on the formation of cerasomes. ${ }^{[24]}$ For example, hydrolysis of triethoxysilyl head moiety and subsequent condensation among silanol groups are so fast that it is difficult to maintain stable vesicle structure under stronger acidic conditions at $\mathrm{pH}$ 1. Under neutral $\mathrm{pH}$ conditions, the hydrolysis of the head group is too slow to form corresponding amphiphilic molecules. Basic conditions seem to be unsuitable for the fabrication of cerasomes owing to the hydrolysis proceed heterogeneously. Thus, stable cerasome dispersion is obtained only under moderate acidic conditions. In addition, this method is applicable to cerasome-forming-lipids that are insoluble in water, such as CFL 1-3 and 6-7. For lipids that are amphiphilic molecules themselves like CFL $\mathbf{4}$ and $\mathbf{5}$, cerasomes can be formed directly through this dispersion method without hydrolysis process. $^{[22]}$

\section{Ethanol sol injection method}

Katigiri et al. ${ }^{[24]}$ introduced ethanol sol injection method to overcome the limitations of $\mathrm{pH}$ conditions on the preparation of cerasomes by ultrasonic dispersion method. The cerasome-forming lipids were incubated in acidic ethanol solution for an appropriate time to promote the hydrolysis of lipids. The sol thus obtained was slowly injected into deionized water with an appropriate $\mathrm{pH}$, followed by ultrasound for a period of time. The prepared cerasomes were stored at room temperature for additional $24 \mathrm{~h}$ to allow the formation of siloxane network.

The ethanol sol injection method has a great advantage for the fabrication of cerasomes in a wide $\mathrm{pH}$ range because the hydrolysis process and the subsequent self-assembling process proceeds independently. ${ }^{[22]}$

\section{Thin film hydration method}

Recently, thin film hydration method has been used for the preparation of cerasomes. ${ }^{[28]}$ Briefly, ceresome-forming-lipids were incubated with acidic ethanol at $\mathrm{pH} 3.0$ overnight at room temperature and then dissolved in chloroform. A lipid thin film was obtained by removal of the organic solvent in a rotary evaporator. The resulted lipid film was further dried in a vacuum oven overnight to completely remove the solvent. The dried lipid film was hydrated in deionized water, followed by vortex mix for a period of time to form multilamellar vesicles. The vesicle dispersion was further ultrasonicated with a probe-type sonicator, resulting in the formation of cerasomes with smaller size. 


\section{Properties of cerasomes as drug carriers}

\section{Morphology}

The morphological structures of cerasomes are mainly obserbed by transmission electron microscopy (TEM) and scanning electron microscopy (SEM). The electron micrographs in Figure 3 indicated that the PTX-loaded cerasomes prepared from CFL 1 through thin film hydration method were spherical and the vesicular size was around $200 \mathrm{~nm}$, which was well consistent with the result (205.2 $28.6 \mathrm{~nm}$ ) as evaluated by dynamic light scattering (DLS) measurements. ${ }^{[27]}$ It is noteworthy that conventional liposomes formed with phospholipids are difficult to characterize with SEM due to the poor structural integrity of the samples.

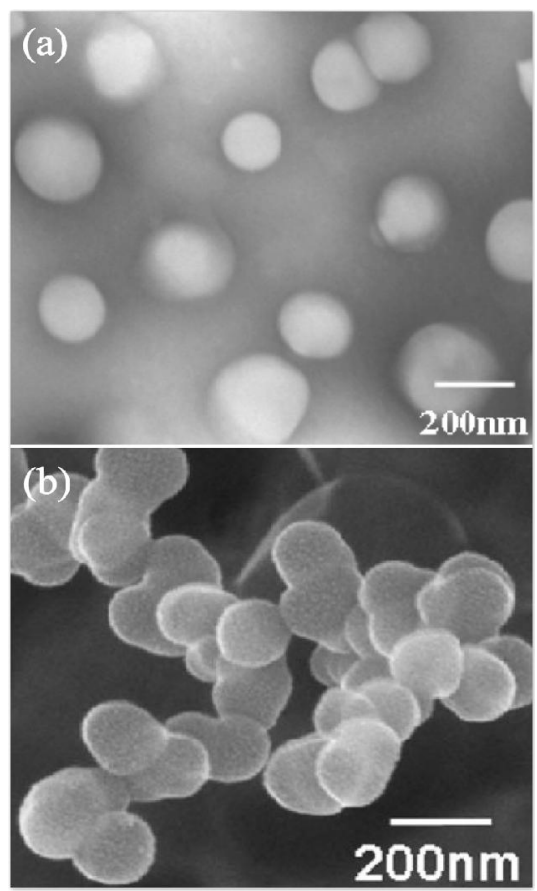

Figure 3 TEM (a) and SEM (b) micrograph of the paclitaxel-loaded cerasomes. ${ }^{[27]}$

One of the most significant features of cerasomes is the presence of siloxane framework on the surface. FT-IR spectroscopy is the primary means to prove the formation of siloxane bonds on the cerasomes surface. Stretching bands attributed to the Si-O-Si and Si-OH groups were observed around 1100 and $920 \mathrm{~cm}^{-1}$, respectively, thus confirming the hydrolysis of triethoxysilyl groups of the CFL, as well as subsequent formation of polysiloxane network.

\section{Stability}

Despite many advantages, conventional liposomes as drug carriers are prone to aggregation and fusion to form large particles, leading to rapid clearance of the vesicles from circulation as well as premature leakage. ${ }^{[31]}$ The polyorganosiloxane network on the surface endows cerasomes remarkably high stability towards surfactant solubilization, long-term storage, as well as in acidic and alkaline conditions, which are impossible for conventional liposomes.

Surfactant solubilization is an important method for evaluating the morphological stability of cerasomes. It was found that cationic liposomes lose the light scattering upon the addition of five equivalents of Triton X-100 (Figure 4a), indicating the complete destruction of the vesicular structure. ${ }^{[22]}$ This phenomenon was very common for liposomes. On the contrary, the light scattering intensity of cerasomes had almost no significant change under the effect of 30 equiv. of Triton X-100, reflecting that cerasomes display excellent stability toward surfactant. It is worth noting that cerasomes could still maintain perfect stability toward Triton X-100 even if a certain proportion of the phospholipid component was incorporated into the vesicle composition. ${ }^{[32]}$

As known to us all, there is an increase in particle size for conventional liposomes because of the aggregation and fusion of broken liposomal vesicles during the storage. However, after storage at $4{ }^{\circ} \mathrm{C}$ for $3 \mathrm{~m}$, PTX-loaded cerasomes had almost no change in particle size (Figure $4 \mathrm{~b}$ ), reflecting higher long-term storage stability of cerasomes than that of liposomes. ${ }^{[33]}$ TEM observations further confirmed that cerasome vesicles still kept spherical structure and were well dispersed. Meanwhile, after place at room tempreture for $3 \mathrm{~d}$, there was no obvious change of particle size for PTX-loaded cerasomes as well. Besides, the size of the freshly prepared cerasomes increased sharply under acidic conditions $(\mathrm{pH}=2)$, indicating that the structure of the vesicles was destroyed. However, the cerasomes prepared after $24 \mathrm{~h}$ displayed remarkable resistance towards both acidic $(\mathrm{pH}=2)$ and alkaline $(\mathrm{pH}=9)$ conditions due to the well-developed siloxane network, which would destabilize conventional liposomes. Moreover, the zeta potentials of cerasomes prepared from CFL 1 was $-30.42 \pm 0.71 \mathrm{mV}$, most likely due to the presence of silanol groups on the outer surface of cerasomes, indicating that the hydrolyzed triethoxysilyl groups were partially polymerized, which imparted cerasomes good morphological stability. ${ }^{[28]}$
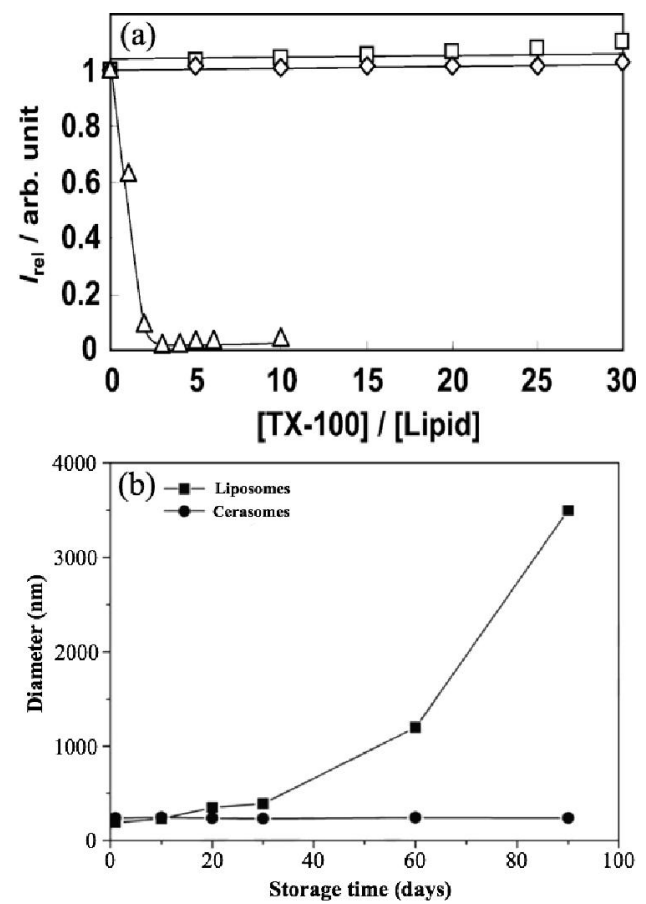

Figure 4 Effect of addition of TX-100 on the light scattering intensity of the cerasomes prepared by CFL $2(\square)$ and $3(\diamond)$ and the liposome $(\triangle)(\mathrm{a}){ }^{[22]}$ Diameters of PTX-loaded liposomes and cerasomes as a function of storage time (b). ${ }^{[33]}$

\section{Biocompatibility}

Cerasomes exhibit better biocompatibility than that of silica nanoparticles owing to the presence of liposomal bilayer membrane provided with a good affinity with cells. Ma et al. ${ }^{[34]}$ investigated the effect of different nanocarriers on the survival rate of normal cell HUVECs. As shown in Figure 5, cells treated with cerasomes showed higher viability than those cultured with silica nanoparticles of similar size at the same concentration level, thus demonstrating that the introduction of liposomal architecture boosts the biocompatibility of cerasomes. Nevertheless, when incubated with cerasomes at a concentration of $0.2 \mathrm{mg} / \mathrm{mL}$ for $24 \mathrm{~h}$, the cell viability for human umbil- 
ical vein endothelial cells (HUVECs) was less than $80 \%$, which was lower than that of conventional 1,2-dipalmityol-sn-glycero-3-phosphocholine (DPPC) liposomes under the same conditions, indicating that the biocompatibility of cerasomes remains to be improved.
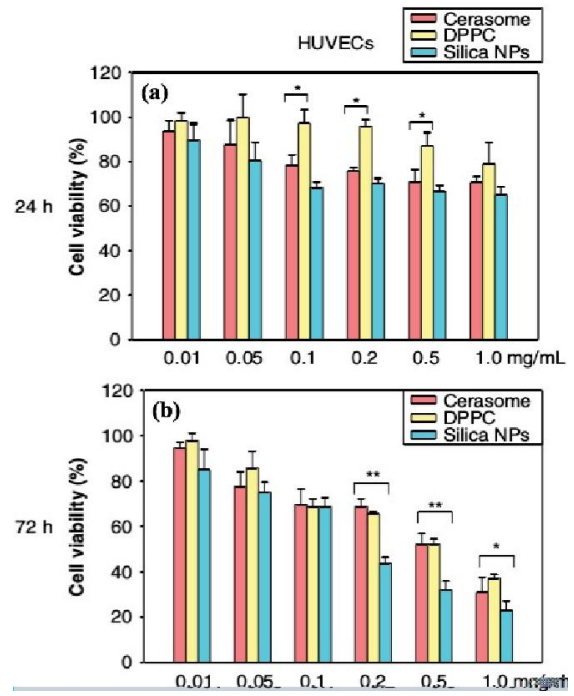

Figure 5 Cell viability of HUVECs cells incubated with varying concentrations of nanoparticles from $0.01-1.0 \mathrm{mg} / \mathrm{mL}$ for $24 \mathrm{~h}$ (a) and $72 \mathrm{~h}(\mathrm{~b}) .^{[34]}$

To solve this problem, cerasome-forming lipids were combined with conventional biocompatible natural lipids to form composite cerasomes via self-assembly method. For example, Cao et al. ${ }^{[32]}$ prepared successfully composite cerasomes from mixtures of cerasomesforming lipids and 1,2-distearoyl-sn-glycero-3-phosphocholine (DSPC) lipids. As shown in Figure 6, when the percentage content of DSPC lipids was $50 \%$, the biocompatibility of cerasomes was significantly improved, close to that of the DSPC liposomes. Meanwhile, the alteration in vesicle composition had no obvious influence on the morphological stability of cerasomes. Therefore, this method opens a door for enhancing the biocompatibility of cerasomes by the introduction of phospholipids with a certain percentage into vesicles without affecting the stability of cerasomes. In addition, a new type of cerasome based on cholesteryl succinyl silane (CSS) with good biocompatibility has been developed recently for the treatment of leukemia. ${ }^{[35]}$

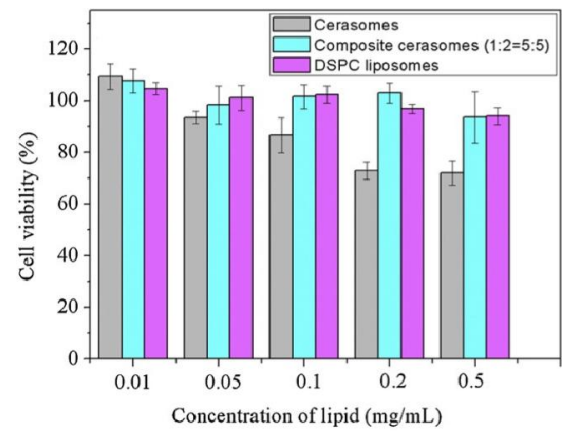

Figure 6 Viability of HUVECs cells after incubating with nanocarriers for $24 \mathrm{~h}^{[32]}$

\section{Drug release behavior}

Liposomal nanohybrid cerasomes can encapsulate both hydrophobic and hydrophilic drugs and the release process can be modulated by regulating the condensation degree and the gap of polyorganosiloxane surface in vivo and in vitro. Cao et al. ${ }^{[27]}$ studied the release of PTX from liposomes and cerasomes. As shown in Figure 7, the drug release from liposomes was much faster than that from ce- rasomes and almost completed within $70 \mathrm{~h}$, while the cerasomes released only $58.2 \%$ within $120 \mathrm{~h}$, thus displaying significantly controlled sustained release behavior. The slower drug release rate for cerasomes might be attributed to the siloxane networks, which limited the flow of the lipid bilayer membrane, resulting in poor membrane permeability and a decrease in drug release rate. Jin et al. ${ }^{[28]}$ examined the release of doxorubicin (DOX) in the cerasomes and made a similar conclusion that cerasomes showed remarkably more sustained release than conventional liposomes. Furthermore, the release profiles of DOX from cerasomes could be readily modulated by altering vesicle composition through DPPG lipids incorporation. With the proportion of dipalmitoylphosphatidylglycerol (DPPG) increasing, the drug release rate and the accumulative release of DOX from cerasomes increased (Figure 8), which might be ascribed to the enhanced membrane permeability as a result of the introduction of DPPG.

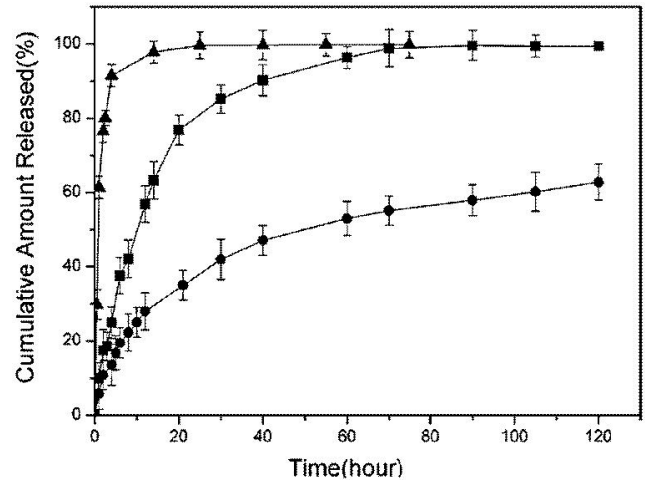

Figure 7 In vitro release of paclitaxel from $\operatorname{PTX}(\boldsymbol{\Delta})$, PTX-loadedliposomes $(\boldsymbol{\square})$ and PTX-loaded-cerasomes $(\bullet)$ into a release medium containing $0.1 \%(V / V)$ of Tween 80 at $37{ }^{\circ} \mathrm{C} .{ }^{[27]}$

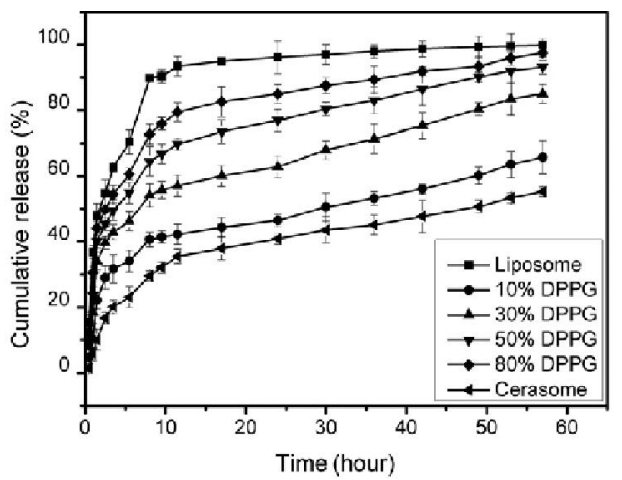

Figure 8 Influences of vesicle composition on the sustained release of DOX from DPPG-cerasomes. ${ }^{[28]}$

Meanwhile, the drug release rate from cerasomes could be precisely controlled by artificially tuning the chemical structure of cerasomes-forming lipids, such as adjusting the numbers of triethoxysilane headgroups and hydrophobic alkyl chain (Figure S1 in Supporting Information). ${ }^{[36]}$ In addition, external stimuli (i.e, $\mathrm{pH},{ }^{[31]}$ temperature, ${ }^{[37,38]}$ and light ${ }^{[39,40]}$ ) have been exploited to control drug release from cerasomes at the desired location and time. These results demonstrate the potential for cerasomes to serve as effective controlled-release drug carriers.

\section{Applications of cerasomes as drug nanocarriers}

\section{General cerasomes}

General cerasomes refer to those directly prepared from conventional organic-inorganic hybrid lipids without the incorporation of any other phospholipid components or surface modification. Cao 
et $a l .{ }^{[33]}$ prepared general cerasomes containing hydrophobic paclitaxel and hydrophilic doxorubicin by thin film hydration method, respectively. Compared with conventional liposomes, drug-loaded cerasomes had higher chemical and storage stability exhibiting more sustained release, and they could effectively kill cancer cells at the same time. Zhang et al. ${ }^{[31]}$ introduced a novel strategy to develop cerasomal microcapsules with high stability and uniform size. DOX-loaded cerasomal microcapsules, displaying a $\mathrm{pH}$-dependent and sustained DOX release profile in vitro, demonstrated remarkable potency in inhibiting the proliferation of tumor cells.

Zhou et al..$^{[41]}$ synthesized a cerasome-forming lipid containing a cleavable disulfide bond, which was used to fabricate redox-responsive general cerasomes through ethanol injection method (Figure 9). Using doxorubicin as model drug, cerasomes released the payload in a redox responsive way. With the presence of glutathione (GSH) in tumor tissues, the lipid bilayer structure could be destroyed after the breakage of disulfide bond, resulting in the release of cargo. Therefore, redox responsive cerasomes are expected to be an intelligent drug delivery platform for intracellular anticancer drug delivery.

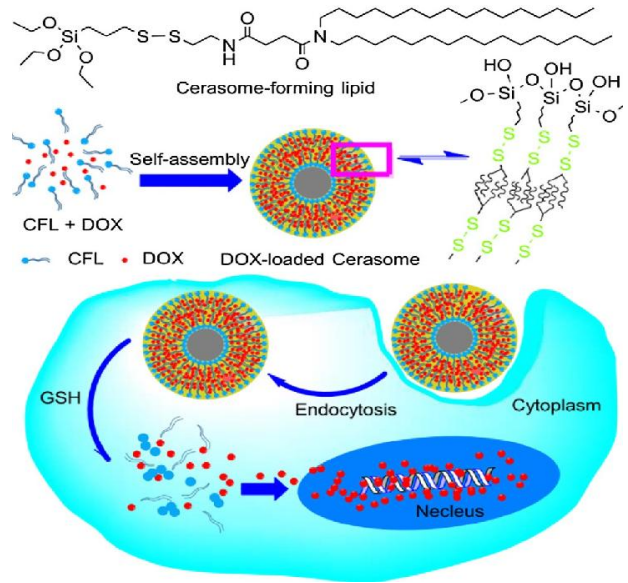

Figure 9 Schematic illustration of the redox responsive cerasomes for intracellular drug delivery. ${ }^{[41]}$

In addition, drug-loaded general cerasomes are usually combined with imaging techniques to construct a nanocarrier system that integrates diagnosis and treatment. As shown in Figure 10, cerasomes with high stability and sustained drug release profile containing $\mathrm{Fe}_{3} \mathrm{O}_{4}$ nanoparticles and the anticancer drug paclitaxel have been developed for simultaneously magnetic targeted drug delivery and magnetic resonance imaging. ${ }^{[42]}$ The utilization of an external magnetic field promoted significantly the internalization of PTX-loaded cerasomes into cancer cells, thereby enhancing the efficacy of the delivered anticancer drug for killing tumor cells.

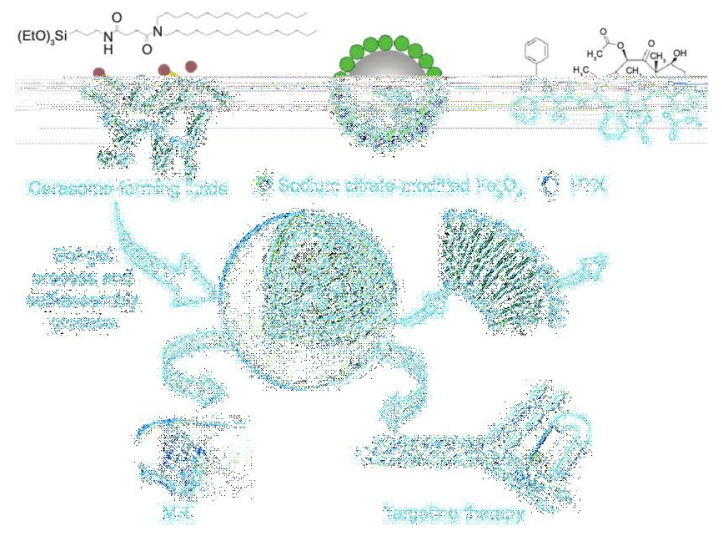

Figure 10 Schematic illustration of PTX-loaded magnetic cerasomes. $^{[42]}$

\section{Composite cerasomes}

Composite cerasomes are usually engineered via incorporating natural phospholipids into cerasomes to further improve their performance such as drug release, biocompatibility, and so on. Wang et al. ${ }^{[26]}$ fabricated cerasomes and composite cerasomes, respectively, from pure cerasome-forming lipids, cerasome-forming lipids and soybean lipids at different molar ratios for loading 10-hydroxycamptothecin (HCPT), both of which exhibited higher structural stability compared with liposomes from pure soybean lipids. Composite cerasomes could reach much higher encapsulation efficiency and drug loading content values for HCPT in comparison with cerasomes. Additionally, composite cerasomes could optimize the release profile of HCPT by the alteration in vesicle compositions, leading to a significantly improved pharmacokinetic profile in vivo. Similarly, Jin et $a l .{ }^{[43]}$ reported the fabrication of composite cerasomes for controlled insulin release by altering vesicle composition through incorporating the phospholipid of DPPC into cerasomes. More importantly, the insulin-loaded composite cerasomes showed a significant and prolonged hypoglycaemic effect compared with liposomes and free insulin. These results demonstrated that composite cerasomes as delivery vehicles have great potential for controlled release of anti-cancer drugs.

Liang et al. ${ }^{[37]}$ developed a new method for cancer treatment by employing high intensity focused ultrasound (HIFU) in combination with thermosensitive composite cerasomes prepared from the incorporation of low-temperature sensitive liposomes including 1,2-dipalmityol-sn-glycero-3-phosphocholine (DPPC), 1,2-distearoyl-snglycero-3-phosphoethanolamine- $N$-PEG-2000 (DSPE-PEG-2000) and 1-stearoyl-2-hydroxy-sn-glycero-3-phosphocholine (MSPC) into cerasomes (Figure 11). Temperature-sensitive cerasomes with remarkable stability, longer blood circulation time and controlled release were successfully fabricated through varying the vesicle composition. The cerasomes could release most of the drugs in only 1 min upon HIFU sonication, which suppressed significantly the growth of tumor bearing mice. This method could quickly release high concentrations of drugs at desired areas, significantly enhancing therapeutic efficacy and avoiding damage to non-treated areas, which holds great potential for efficient local chemotherapy of cancer.

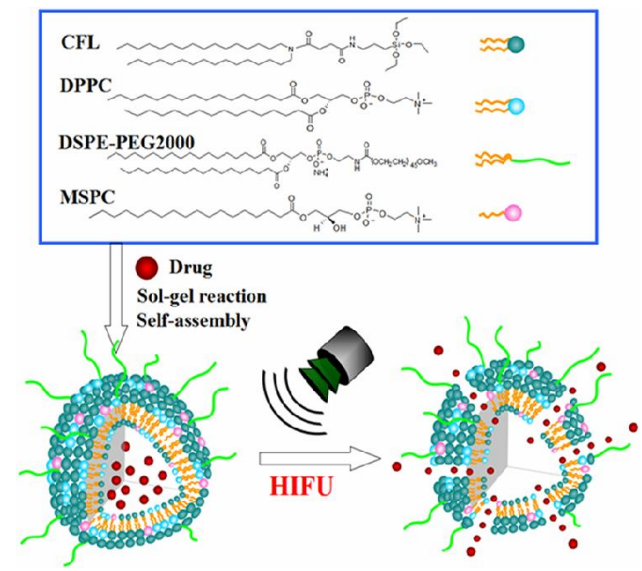

Figure 11 Schematic illustration for the formation of drug-loaded thermosensitive cerasomes and the drug release upon HIFU sonication. ${ }^{[37]}$

\section{Functionalized cerasomes}

As is known to us all, it takes a long time for drug-loaded liposomes to accumulate in tumor tissues via the enhanced permeability and retention (EPR) effect, inevitably causing harm to normal tissues due to the morphological instability. Compared with liposomes, the presence of silanol groups on the surface enables cerasomes to be 
functionalized with biomolecules that are specific to tumor cells to selectively deliver drugs to cancer cells with an improved targeting capability. For example, Wang et al. ${ }^{[44]}$ reported the fabrication of triphosphonium (TPP) surface-modified cerasomes for mitochondrial-targeted transport of anticancer drug DOX (Figure 12). TPP functionalized cerasomes could easily penetrate the mitochondrial membrane and accumulate within the mitochondrial matrix owing to the completely opposite charges between TPP and mitochondrial membrane. Compared with non-targeted cerasomes, TPP targeted cerasomes exhibited higher drug accumulation in mitochondria, resulting in more pronounced anticancer effects.

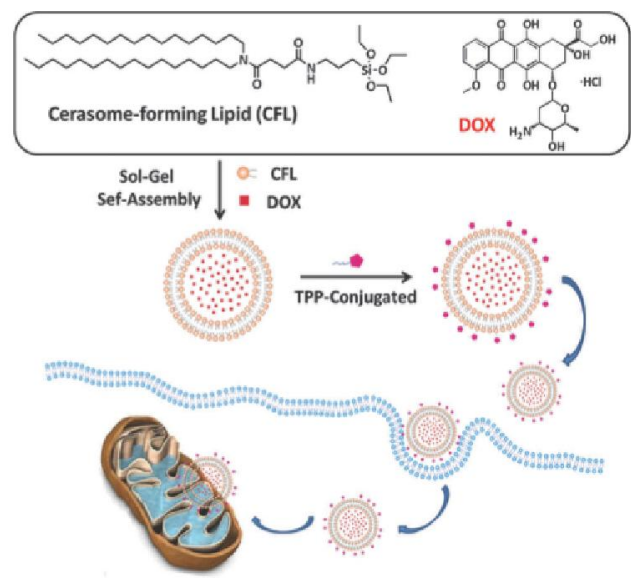

Figure 12 Schematic illustration of the formation process of the DOX-loaded cerasomes and TPP modified cerasomes. ${ }^{[4]}$

Analogously, Leung et al. ${ }^{[45]}$ synthesized functionalized cerasomes by employing the chemical conjugation of anti-epidermal growth factor receptor monoclonal antibodies (anti-EGFR mAbs) to cerasomes for the selective delivery of therapeutic/imaging agents to cancer cells. Anti-EGFR-conjugated cerasomes could selectively target tumor cells that express EGFR receptor on the surface, and the cellular uptake of functionalized cerasomes increased with the membrane expression of EGFR by cancer cells increasing.

Besides, the surface of cerasomes could be functionalized with magnetic metal alloy by using electroless plating to create magnetic cerasomes. The magnetic cerasomes with unique magnetic properties were capable of manipulation by a magnet, having great potential to perform as ideal drug carriers for selective targeting with external magnetic fields. ${ }^{[46]}$

Therefore, the combination of functionalized cerasomes with anticancer drugs is expected to construct a targeted drug carrier system for the delivery of drug molecules to desird location.

\section{Novel cerasomes}

In recent years, with the further study of chemical structure associated with organic-inorganic hybrid lipids, several novel types of liposomal nanohybrid cerasomes have been developed for diverse application demands.

As shown in Figure 13, a newly introduced cerasome with remarkably high stability was developed based on cholesteryl succinyl silane (CSS) through ethanol injection method by Ma et al. ${ }^{[35]}$ It's worth noting that the CSS cerasomes alone could selectively inhibit the proliferation of leukemia cells without damaging normal blood cells. More significantly, DOX-loaded CSS cerasomes exhibited improved anticancer efficiency of cancer therapeutics in comparison with pure DOX. This method makes CSS cerasome a potential nanocarrier for the treatment of leukemia due to the maximized efficacy and minimized side effects. They prepared a multifunctional drug-delivery platform from CSS containing DOX and $\mathrm{Fe}_{3} \mathrm{O}_{4}$ magnetic nanoparticles in combination with the modification of gold nanoshells on the surface (Figure 14). ${ }^{[47]}$ This multifunctional CSS cerasomes that integrated magnetic resonance imaging (MRI), magnetic-targeted drug delivery, NIR light-triggered drug release, and photothermal therapy could effectively kill cancer cells with remarkably enhanced efficacy, showing broad application prospects for the combined theranostics of cancer. Afterwards, Jing et al ${ }^{[48]}$ successfully fabricated ${ }^{177} \mathrm{Lu}$-labeled CSS cerasomes encapsulating indocyanine green (ICG) from the mixture of CSS and 1,2-distearoyl-snglycero-3-phosphoethanol-amine- $N$-[carboxy(polyethylene glycol)2000]-1,4,7,10-tetraaza-cyclododecane-1,4,7,10-tetraacetic acid monoamide (DSPE-PEG2000- DOTA) through ethanol injection method, followed by chelating radioisotope of ${ }^{177} \mathrm{Lu}$ for NIR fluorescence/nuclear imaging and photothermal ablation of cancer. The results demonstrated that CSS vesicles could selectively accumulate in tumor site, which could effectively ablate tumor via photothermal effects. This approach would foster an innovative avenue for the development of cerasome-based therapeutic agents.

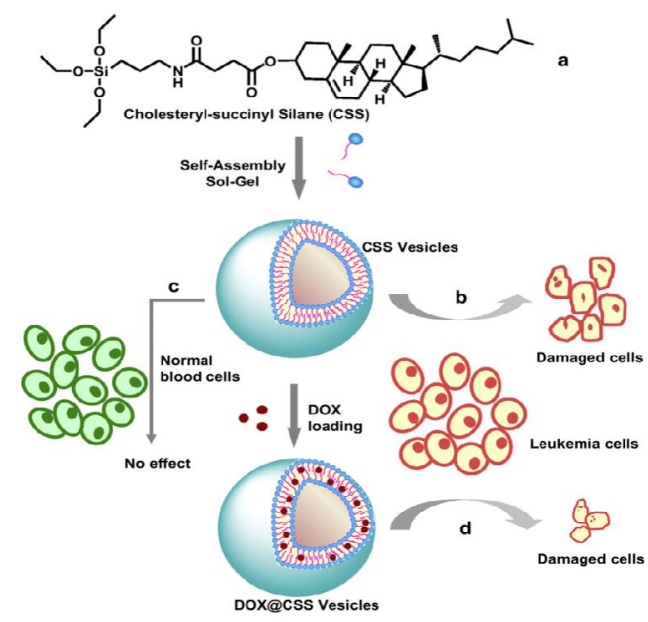

Figure 13 Schematic antiproliferative effects of nanohybrid vesicles of CSS toward leukemia cells. ${ }^{[35]}$

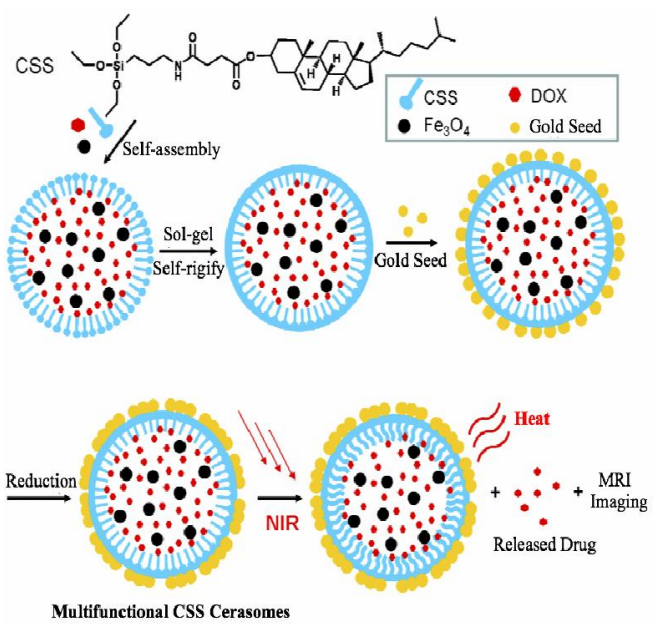

Figure 14 Schematic illustration of multifunctional CSS nanomicelles. $^{[47]}$

Photodynamic therapy (PDT) is an emerging method for cancer therapy, but most photosensitizers used clinically are prone to aggregation in aqueous solutions, greatly reducing the photosensitizing efficiency. To solve this problem, Liang et al ${ }^{[49]}$ reported the fabrication of cerasomal photosensitizers via the conjugation of porphyrins to nanohybrid cerasomes (Figure 15). The alternating arrangement of porphyrins in the lipid bilayer could prevent them from aggregation, 
and the chemical bonding can effectively avoid the premature leakage of photosensitizers during systemic circulation, thus significantly improving the efficacy of PDT. Besides, porphyrin bilayer cerasomes could be efficiently internalized into cancer cells and exhibit significant phototoxicity. More importantly, the capability to encapsulate drug molecules in the internal aqueous core of porphyrin conjugated cerasome made it a promising nanocarrier for the therapy of cancer by employing chemotherapy in combination with PDT simultaneously.

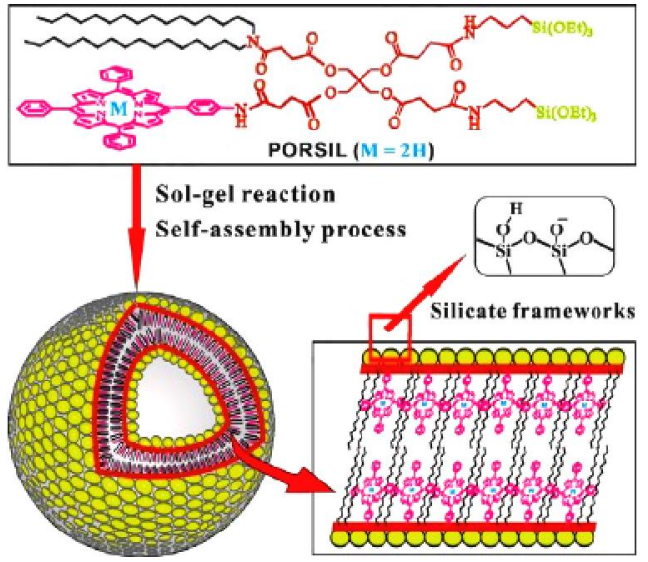

Figure 15 Schematic illustration of porphyrins-conjugated cerasomes. ${ }^{[49]}$

\section{Organic-inorganic hybrid bicelles}

Recently, Kikuchi et al. constructed a new type of organic-inorganic hybrid bicelles (HBs) by the mixture of long-chain organoalkoxysilane lipids (CFL) and short-chain phospholipids. ${ }^{[50,51]}$ Such hybrid bicelles have received widespread attention owing to the presence of the polyorganosiloxane surface, which offers remarkably higher stability than conventional phospholipid bicelles. ${ }^{[50,52]}$ Moreover, such disc-like nanoparticles exhibit preferential cellular uptake and higher microvascular adhesion than spherical nanoparticles. ${ }^{[53-57]}$ In addition, hybrid bicelles are capable of encapusulating hydrophobic drugs. Therefore, hybrid bicelles are expected to be novel promising nanocarriers for the delivery of hydrophobic drugs.

Lin et al. reported the formation of hydrophobic doxorubicin loaded hybrid bicelles generated from CFL and short phospholipids DHPC at the ratio of 7:2 via traditional thin film hydration method (Figure 16) ${ }^{[52]}$ It was found that the disc-like bicelles displayed similar significantly high stability to cerasome vesicles, but they had higher cellular uptake than spherical cerasomes. More importantly, drug-loaded bicelles exhibited $\mathrm{pH}$-sensitive release behavior and could effectively inhibit the tumor growth. They developed PEGylated nanodiscs by incorporating PEGylated phospholipids with different molar percentages into the hybrid bicelles to modulate drug release rate. The results showed that the drug release rate of DOX from hybrid bicelles increased with increasing the molar percentages of DSPE-PEG2000. ${ }^{[59]}$ Afterwards, they fabricated multifunctional hybrid bicelles co-encapsulating hydrophobic doxorubicin (HDOX) and indocyanine green (ICG) for synergetic chemo/photothermal therapy (Figure 17). ${ }^{[58]}$ The drug-loaded bicelles showed temperature-sensitive drug release behavior and remarkable photothermal cytotoxicity. Such bicelles could selectively accumulate at the tumor site with enhanced therapeutic efficacy and minimal side effects. Thus, this multifunctional bicelles can perform as promising drug delivery platform for efficient chemo/photothermal therapy of cancer simultaneously.

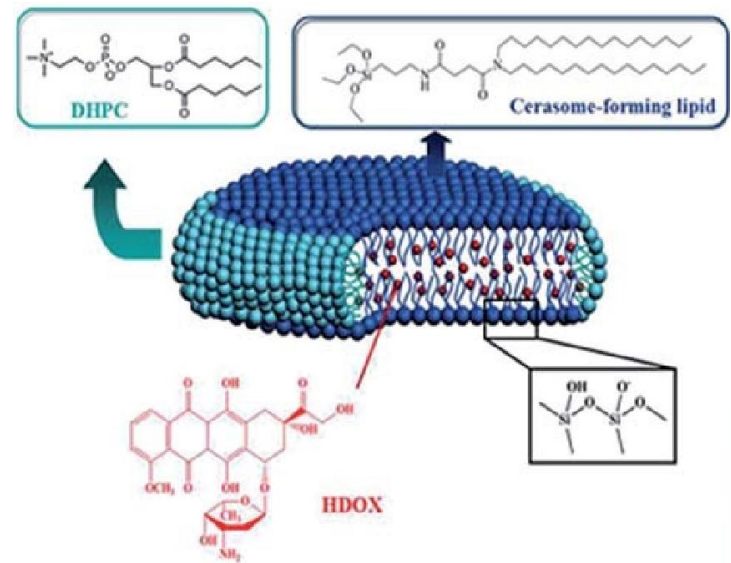

Figure 16 Schematic illustration of hydrophobic doxorubicin loaded hybrid bicelles. ${ }^{[52]}$

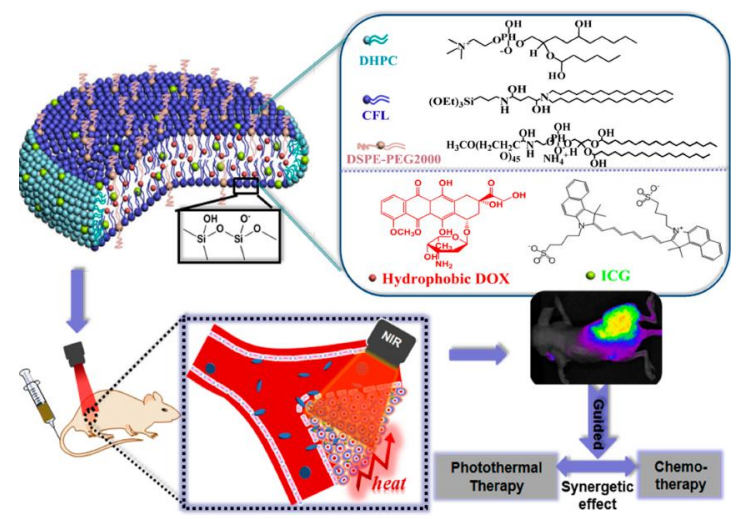

Figure 17 Schematic representation of DOX/ICG loaded hybrid bicelles for fluorescence imaging guided chemo/photothermal therapy of cancer. ${ }^{[58]}$

\section{Conclusion}

Liposomal hybrid cerasomes have made significant progress as novel drug delivery nanocarriers in the field of biomedical medicine, especially in cancer diagnosis and treatment due to many excellent advantages. Nevertheless, there are still several drawbacks that limit the further development of cerasomes. Although it is essential for drug-carriers to possess high stability, the slow rates of drug release from such stable cerasomes make it difficult to maintain an ideal concentration at the desired site, which limit the efficacy in cancer treatment. Therefore, external stimuli (i.e., $\mathrm{pH}$, temperature, and light) may be utilized to trigger a rapid drug release from cerasomes to enhance drug accumulation in tumor tissues and cells. Besides, it is difficult to control the size distribution through conventional methods for cerasomes preparation, which has significant impact on the pharmacokinetics in vivo. With the aim to obtain cerasomes with uniform size distribution, the extrusion method may be used after the preparation of multilamellar cerasomes. In addition, most of the researches on cerasomes at present are focused on in vitro, and the studies in vivo are much more complicated. The long-term biocompatibility and pharmacokinetic behavior of cerasomes in vivo should be further studied systematically. In order to solve the problems above, joint efforts and collaborations of scientific workers are required to realize clinical applications of cerasomes as drug delivery systems as soon as possible. 


\section{Acknowledgement}

The work is supported by the Research Project of the Shanghai Municipal Science and Technology Commission (No. 17441904800).

\section{References}

[1] Kessler, D. A.; Austin, R. H.; Levine, H. Cancer Res. 2014, 74, 4663.

[2] Allen, T. M.; Cullis, P. R. Science 2004, 303, 1818.

[3] Bamrungsap, S.; Zhao, Z.; Chen, T.; Wang, L.; Li, C.; Fu, T.; Tan, W. Nanomedicine 2012, 7, 1253.

[4] Bonifácio, B. V.; Silva, P. B. D.; Negri, K. M. S.; Bauab, T. M.; Chorilli, M. Int. J. Nanomed. 2014, 9, 1.

[5] Kingsley, J. D.; Dou, H.; Morehead, J.; Rabinow, B.; Gendelman, H. E.; Destache, C. J. J. Neuroimmune Pharm. 2006, 1, 340.

[6] Tiwari, G.; Tiwari, R.; Sriwastawa, B.; Bhati, L.; Pandey, S.; Pandey, P.; Bannerjee, S. K. Int. J. Pharm. Investig. 2012, $2,2$.

[7] Seleci, M.; Ag, S. D.; Scheper, T.; Stahl, F. Int. J. Mol. Sci. 2017, 18, 7.

[8] Zhang, Y.; Zhai, M.; Chen, Z.; Han, X.; Yu, F.; Li, Z.; Xie, X.; Han, C.; Yu, L.; Yang, Y. Drug Deliv. 2017, 24, 1045.

[9] Li, S.; Goins, B.; Zhang, L.; Bao, A. Bioconjugate Chem. 2015, 23, 1322.

[10] Jing-Hai, M. U.; Zhou, Y. E.; Zhang, Q.; Yin, J. Curr. Biotechnol. 2016, 6, 179.

[11] Anna, W.; Juergen, B. Nanomaterials 2017, 7, 189.

[12] Salonen, J.; Kaukonen, A. M.; Hirvonen, J.; Lehto, V. P. J. Pharm. Sci. 2008, 97, 632 .

[13] Zhao, H.; Duong, H. H. P.; Yung, L. Y. L. Macromol. Rapid Commun. 2010, 31, 1163

[14] Kamimura, M.; Nagasaki, Y. J. Photopolym. Sci. Technol. 2013, 26, 161.

[15] Biswas, S.; Kumari, P.; Lakhani, P. M.; Ghosh, B. Eur. J. Pharm. Sci. 2016, 83, 184.

[16] Pal, K.; Banthia, A. K.; Majumdar, D. K. J. Mater. Sci.: Mater. Med. 2014, 25, 2215.

[17] Bastiancich, C.; Danhier, P.; Préat, V.; Danhier, F. J. Control. Release 2016, 243, 29.

[18] Lasic, D. D. J. Control. Release 1997, 48, 203.

[19] Lee, S. M.; Chen, H.; Dettmer, C. M.; O'Halloran, T. V.; Nguyen, S. T. J. Am. Chem. Soc. 2007, 129, 15096.

[20] Leung, S. L.; Zha, Z. B.; Teng, W. B.; Cohn, C.; Dai, Z. F.; Wu, X. Y. Soft Matter 2012, 8, 5756.

[21] Katagiri, K.; Ariga, K.; Kikuchi, J. I. Cheminform 1999, 30, 661.

[22] Hashizume, M.; Kawanami, S. I.; Iwamoto, S.; Isomoto, T.; Kikuchi, J. I. Thin Solid Films 2003, 438, 20.

[23] Liang, X. L.; Li, X. D.; Jing, L. J.; Xue, P.; Jiang, L. D.; Ren, Q. S.; Dai, Z. F. Chemistry 2013, 19, 16113.

[24] Katagiri, K.; Hamasaki, R.; Ariga, K.; Kikuchi, J. I. J. Sol-Gel Sci. Technol. 2003, 26, 393.

[25] Kikuchi, J. I.; Yasuhara, K. Adv. Biomimetics 2011, 2, 232.

[26] Wang, Y.; Chen, Y.; Zhang, M. Z.; Qu, H.; Zheng, J.; Pang, Q.; Yan, X. RSC Adv. 2016, 6, 16292.

[27] Cao, Z.; Ma, Y.; Yue, X. L.; Li, S.; Dai, Z. F.; Kikuchi, J. Chem. Commun. 2010, 46, 5265.

[28] Jin, Y. S.; Yue, X. L.; Zhang, Q. Y.; Wu, X.; Cao, Z.; Dai, Z. F. Acta Biomater. 2012, 8, 3372.

[29] Katagiri, K.; Hashizume, M.; Ariga, K.; Terashima, T.; Kikuchi, J. Chemistry 2007, 13, 5272.

[30] Li, S.; Ma, Y.; Yue, X.; Cao, Z.; Liu, S.; Dai, Z. J. Disper. Sci. Technol. 2010, 31, 1727.
[31] Zhang, C. Y.; Cao, Z.; Zhu, W. J.; Liu, J.; Jiang, Q.; Shuai, X. T. Colloids Surface B 2014, 116, 327.

[32] Cao, Z.; Yue, X. L.; Jin, Y. S.; Wu, X.; Dai, Z. F. Colloids Surface B 2012 , $98,97$.

[33] Cao, Z. Ph.D. Dissertation, Harbin Institute of Technology, Harbin, 2011 (in Chinese).

[34] Ma, Y.; Dai, Z. F.; Gao, Y. G.; Cao, Z.; Zha, Z.; Yue, X.; Kikuchi, J. Nanotoxicology 2011, 5, 622 .

[35] Ma, Y.; Dai, Z. F.; Zha, Z. B.; Gao, Y.; Yue, X. Biomaterials 2011, 32, 9300 .

[36] Liang, X. L.; Li, X. D.; Jing, L. J.; Xue, P.; Jiang, L.; Ren, Q.; Dai, Z. F. Chemistry 2013, 19, 16113.

[37] Liang, X. L.; Gao, J.; Jiang, L. D.; Luo, J.; Jing, L.; Li, X.; Jin, Y.; Dai, Z. F. ACS Nano 2015, 9, 1280.

[38] Su, Q.; Pu, X.; Bai, H.; Chen, X.; Liao, X.; Huang, Z.; Yin, G. Curr. Drug Deliv. 2017, 14, 1567.

[39] Liang, X. L.; Yue, X. L.; Dai, Z. F.; Kikuchi, J. Chem. Commun. 2011, 47, 4751.

[40] Liang, X. L.; Li, X.; Yue, X.; Dai, Z. F. Angew. Chem., Int. Ed. 2011, 50, 11622.

[41] Zhou, G.; Li, L.; Xing, J.; Jalde, S.; Li, Y.; Cai, J.; Chen, J.; Liu, P.; Gu, N.; Ji, M. Colloids Surface B 2016, 148, 518.

[42] Cao, Z.; Zhu, W. J.; Wang, W.; Zhang, C.; Xu, M.; Liu, J.; Feng, S. T.; Jiang, Q.; Xie, X. Int. J. Nanomed. 2014, 9, 5103.

[43] Jin, Y. S.; Li, Y. Y.; Pan, H. J.; Dai, Z. F. RSC Adv. 2014, 4, 42808.

[44] Wang, Y. F.; Wang, B. B.; Liao, H.; Song, X.; Wu, H.; Wang, H.; Shen, H.; Ma, X.; Tan, M. J. Mater. Chem. B 2015, 3, 7291.

[45] Leung, S. L.; Zha, Z.; Cohn, C.; Dai, Z. F.; Wu, X. Colloids Surface B 2014, 121, 141.

[46] Minamida, D.; Okada, S.; Hashizume, M.; Sasaki, Y.; Kikuchi, J.-i.; Hosoito, N.; Imori, T. J. Sol-Gel Sci. Technol. 2008, 48, 95.

[47] Ma, Y.; Liang, X. L.; Tong, S.; Bao, G.; Ren, Q. S.; Dai, Z. F. Adv. Funct. Mater. 2013, 23, 815.

[48] Jing, L. J.; Shi, J. Y.; Fan, D.; Li, Y.; Liu, R.; Dai, Z.; Wang, F.; Tian, J. ACS Appl. Mater. Interfaces 2015, 7, 22095.

[49] Liang, X. L.; Li, X. D.; Yue, X. L.; Dai, Z. F. Angew. Chem., Int. Ed. Engl. 2011, 50, 11622.

[50] Yasuhara, K.; Miki, S.; Nakazono, H.; Ohta, A.; Kikuchi, J. Chem. Commun. 2011, 47, 4691.

[51] Yasuhara, K.; Hayashi, H.; Jun-Ichi, K. Chem. Lett. 2012, 41, 1223.

[52] Lin, L.; Wang, X.; Guo, Y.; Ren, K.; Li, X.; Jing, L.; Yue, X.; Zhang, Q.; Dai, Z. RSC Adv. 2016, 6.

[53] Rodríguez, G.; Barbosabarros, L.; Rubio, L.; Cócera, M.; Lópeziglesias, C.; De, 1. M. A.; López, O. Colloids Surfaces B 2011, 84, 390.

[54] Barua, S.; Yoo, J. W.; Kolhar, P.; Wakankar, A.; Gokarn, Y. R.; Mitragotri, S. P. Natl. Acad. Sci. USA 2013, 110, 3270.

[55] Zhang, W.; Sun, J.; Liu, Y.; Tao, M.; Ai, X.; Su, X.; Cai, C.; Tang, Y.; Feng, Z.; Yan, X. Mol. Pharm. 2014, 11, 3279.

[56] Doshi, N.; Prabhakarpandian, B.; Rea-Ramsey, A.; Pant, K.; Sundaram, S.; Mitragotri, S. J. Control. Release 2010, 146, 196.

[57] Adriani, G.; Tullio, M. D. D.; Ferrari, M.; Hussain, F.; Pascazio, G.; Liu, X.; Decuzzi, P. Biomaterials 2012, 33, 5504.

[58] Li, L.; Liang, X.; Xu, Y.; Yang, Y.; Li, X.; Dai, Z. Bioconjugate Chem. 2017, 28, 2410.

[59] Lin, L.; Wang, X.; Li, X.; Yang, Y.; Yue, X.; Zhang, Q.; Dai, Z. Bioconjugate Chem. 2016, 28, 53. 